\title{
Piracy and Quality Choice in Monopolistic Markets
}

\author{
Matteo A Ivisi \\ Department of E conomics, University of Bologna \\ Elena Argentesi \\ European University Institute, Florence \\ E manuela Carbonara \\ Department of E conomics, University of Bologna
}

A pril 2002

\begin{abstract}
A bstract
We study the impact of piracy on the quality choices of a monopolist. In the absence of piracy, the monopolist has no incentive to dixerentiate its products. With piracy the monopolist might instead produce more than one quality, so that dixerentiation arises as the optimal strategy. This is because the producer wants to divert consumers from the pirated good to the original one. Dixerentiation involves either producing a new, low-quality good such that piracy is either eliminated or still observed in equilibrium.

J EL Classi..cation Numbers: L12, L 15, L82, L86.

K eywords: Product dixerentiation, Multiproduct monopolist, Quality, Piracy.

\footnotetext{
${ }^{x}$ W e are grateful to $M$ assimo $M$ otta for comments, discussions and advice, as well as to G iacomo Calzolari, Vincenzo Denicolò, Paolo Garella and Luca L ambertini for comments and suggestions.
} 


\section{Introduction}

The huge increase of piracy and private copying is a phenomenon that in recent years has greatly axected the market for information goods, and speci... cally digital goods such as software and music compact disks. The widespread practice of ..le-sharing through the Internet, together with the improvements and the greater availability of copying technologies ( like peer-to-peer connections), lead to a boom of unauthorized copies.

The reproduction of digital information dixers from the reproduction of non-digital media such as journals, books, audio and video cassettes. Digital media are characterized by a "vertical" pattern of reproduction, ${ }^{1}$ since it is enough to have an original and then copies can be made out of copies without a progressive decline in their quality. Therefore in markets for digital goods the traditional result of "indirect appropriability" cannot apply. ${ }^{2}$ W ith indirect appropriability the seller can extract the rents from all users by charging a higher price for the original since their total willingness to pay as a whole is higher than the single buyer's. When quality does not decline with the number of copies the price that extracts all surplus from all users would be too high to be axordable by the single buyer. These features enhance the harmful impact of piracy on pro..ts. ${ }^{3}$

From a legal perspective, one way in which ..rms can counteract this phenomenon is by undertaking legal actions against the infringements of copyright laws (the most recent case is the suit brought by the ...ve major record companies against Napster) and by lobbying for stricter copyright laws. ${ }^{4}$ The goal of our paper is instead to examine from an economic perspective the impact of piracy on the business strategies of a ..rm. ${ }^{5}$

We study how piracy axects the ..rm's incentives to vertically dixerentiate the quality of its products. In general the presence of piracy reduces the demand for the original good and therefore pro..ts. The monopolist could then introduce a low-quality good in order to capture at least part of the demand for piracy, shifting it from the illegal market, where copies of the

\footnotetext{
${ }^{1} \mathrm{~F}$ or a classi..cation and description of information reproduction, see Shy (2001).

${ }^{2} \mathrm{~T}$ his concept was ..rst introduced by Liebowitz (1985).

${ }^{3} \mathrm{~A}$ s pointed out by Shy and Thisse (1999), the negative impact of copying on producers' pro..ts can be mitigated by the presence of network externalities, i:e: when consumers' utility increases in the number of other consumers using the good (either bought or copied). This assumption is quite plausible with respect to software but it is hardly applicable to other information goods such as music and printed items.

${ }^{4} \mathrm{~F}$ or instance, the European Union has recently issued a directive on copyright protection of online digital goods (Directive 2001/29/CE).

${ }^{5}$ We consider a situation where piracy cannot be completely eliminated by copyright protection laws.
} 
high-quality good are exchanged.

In practice, producing a new, low-quality good means that the ..rm introduces a reduced version of what was previously produced without some of its features. Examples of vertical dixerentiation in the software market are the reduced versions released as shareware or freeware on the Internet. In classical music, lower-quality (and lower-cost) CDs are sold without a booklet and performed by less famous orchestras. Similarly, music companies are developing technologies to sell music on-line ${ }^{6}$ (without package and without a booklet with photos, lyrics and information on the artists) as a way to capture consumers with low willingness to pay who could otherwise be potential pirates.

In our model, the goods produced by the monopolist can be obtained in two dixerent ways: consumers can either purchase them in the original version or pay for unauthorized copies. Our de. nition of a copy includes both a copy made by somebody else and bought illegally ( $i: e:$ a CD from an unauthorized vendor) and a copy made by the consumer herself ( $i: e:$ a $C D$ copied with a CD burner). Given that we refer mainly to digital goods, we assume that the original good and its copies are identical in terms of quality.

We also assume that consumers are heterogeneous in two respects. First, there is a continuum of consumers identical in tastes but with dixerent preferences for quality. Second, consumers are characterized by two dixerent costs of pirating (or going to the illegal market), under the assumption that a higher cost of copying is associated with a higher willingness to pay for quality.

Our main results are as follows. In the absence of piracy, the monop ol ist has no incentive to dixerentiate its products. With piracy the monopolist might instead produce more than one quality, so that dixerentiation arises as the optimal strategy.

The analysis involves two distinct cases. If the proportion of consumers with high cost of pirating is low and the monopolist dixerentiates its products, prices are set such that the high-cost consumers buy the high-quality good, whereas low-cost consumers will either buy the low-quality good or pirate. In this case, vertical dixerentiation will be more pro..table than no dixerentiation when the heterogeneity of consumers with respect to their cost of pirating is suф ciently high. Here, the monopolist indeed ..ghts pirates through vertical dixerentiation, producing a new and lower quality that eliminates demand for copies and hence piracy. When instead consumers are more similar in their costs of pirating, the monopol ist will ..ght piracy by set-

\footnotetext{
${ }^{6} \mathrm{~T}$ he ...ve $\mathrm{M}$ ajors have recently made two joint ventures to sell music online, namely MusicN et and Pressplay.
} 
ting a price low enough such that everybody buys the good, and no vertical dixerentiation takes place.

If the proportion of high-cost consumers is high and the monopolist produces two goods, prices are set such that high-cost consumers buy both goods. In this situation, an interesting trade-ox in the choice of quality levels arises. The monopolist still has an incentive to oxer a low quality level that is high enough to attract consumers with low willingness to pay. However, if this level is high enough, some high-cost consumers may switch from the high- to the low-quality good. This second exect may counterbalance the incentive to eliminate piracy by increasing the low quality level and, in equilibrium, the monopolist might admit some piracy while still dixerentiating.

Our work builds on the literature on copying and piracy. In particular, it is related to a recent paper by Gayer and Shy (2001) that analyzes the incentives of publishers to distribute via the Internet versions of digital goods that compete with products sold in stores. Under the assumption of network externalities betwen buyers of the original good and "downloaders" from the Internet, they show that the introduction of a product of (exogenously-given) lower quality over the Internet is pro..t-enhancing for the monopolist. The issue of copyright protection in the presence of network externalities is also addressed by Conner and R umelt (1991) and, in an horizontally dixerentiated duopoly, by Shy and Thisse (1999).

One major dixerence of our approach relative to the previous literature is that we endogenize the choice of product quality by making use of a model of vertical dixerentiation. Moreover, we do not assume network externalities between buyers of the original good and "downloaders" from the Internet, which instead drive Gayer and Shy's results. ${ }^{7}$ The previous models showed that the distribution of a lower-quality good over the Internet may be profitable for the producer because it may raise the demand for the original good through demand-side externalities. On the contrary, in our framework the introduction of a lower quality allows the producer to divert some consumers from copying the good to buying an original (lower-quality) version and is therefore a way to reduce (or eliminate) piracy.

Our model is more generally related to the literature on copying (e.g., J ohnson, 1985; Liebowitz, 1985; B esen and K irby, 1989; Varian, 2000). These papers generally assume that copies can be made only from originals ${ }^{8}$ and therefore producers can appropriate some of the consumer surpl us from illegal

\footnotetext{
${ }^{7}$ Indeed, Hui et al. (2001) tested empirically the impact of piracy on the legitimate demand for recorded music and found that the potential positive exects of piracy (network externalities, sampling, sharing, and others), if they exist, do not compen sate for the direct loss of customers.

${ }^{8} \mathrm{~A}$ Iternatively, copies of copies have a lower quality than copies made from originals.
} 
copies. ${ }^{9}$ T herefore, even in the absence of network externalities, copying may be pro..table because of indirect appropriability. Our paper instead rules out any potential bene.cial exect of piracy for producers (either through some kind of appropriability or through network exects) and studies the pricing policies of a monopolist faced with this problem.

Our paper is also an application of the theoretical literature on vertical dixerentiation by a monopolist. The main idea of this literature is that a monopolist may want to segment the market by oxering dixerent qualities of the same good in order to extract more consumer surplus. T wo classes of models investigate dixerent assumptions on qual ity costs. ${ }^{10}$ Gabszewicz et al. (1986), building on the model by Shaked and Sutton (1982), assume ..xed costs of quality improvement and show that the number of products sold depends on the dispersion of consumers' incomes. Mussa and R osen (1982), Itoh (1983), Maskin and Riley (1984), and Lambertini (1987) study instead the case where unit production costs are increasing in quality, and ..nd that the monopolist has an incentive to produce a broad range of qualities in order to segment the market.

Our contribution to this literature is that we propose an alternative explanation for vertical dixerentiation, namely piracy. We do this by focusing on a case where no vertical dixerentiation would arise in an equilibrium without piracy. In this setting, we show that when piracy is instead possible, it may be more pro..table to introduce a lower quality rather than to produce one quality only.

The paper is organized as follows. Section 2 presents the base model. In sections 3 and 4 we analyze the price and quality strategies of a monopolist producing a single product and two products respectively. Section 5 compares the pro.ts of vertical dixerentiation with those resulting from the strategy of a single quality level and derives conditions for the introduction of a lower quality. Section 6 concludes.

\section{The model}

We assume that consumers can obtain the good in two dixerent ways: they can purchase its original version in the legal market or, alternatively, obtain a copy in an illegal market, where only unauthorized copies are available. ${ }^{11}$

\footnotetext{
${ }^{9} \mathrm{~T}$ herefore these models are applicable mainly to non-digital goods.

${ }^{10} \mathrm{~F}$ or a model that considers both types of assumptions on costs in an oligopolistic setting, see M otta (1993).

${ }^{11} \mathrm{~T}$ his is what we label piracy in the rest of the paper, although we disregard copyright issues and related legal punishments.
} 
Examples of goods where illegal copies exist include music CDs, software, videos and also design clothes and accessories.

\subsection{Production}

We consider a monopolist operating in a market where consumers have the possibility of pirating the good (or obtaining a copy on the illegal market). ${ }^{12}$ Piracy reduces demand in the legal market and potentially ..rms' pro..ts. When choosing its business strategies, the monopolist tries to counter this problem. Throughout the paper, we assume that price discrimination for products of the same quality is not an available option for the monopolist (for instance, because arbitrage is possible). However, the monopolist can choose to vertically dixerentiate its goods. The monopolist has two main strategies: it can produce one good only, whose quality and price are $q$ and $p_{\text {; }}$ or two goods, whose qualities are $q_{1}$ and $q_{2}$, with $q_{1}<q_{2} ;$ and $q_{1} 2[0 ;+1)$, $i=1 ; 2$. Let $p_{1}$ denote the price of the good of quality $q_{1}$ and $p_{2}$ the price of qual ity $q_{2}$. We assume that the ..rm has neither variable production costs nor quality-dependent (..xed) costs. ${ }^{13}$

We are looking at a three-stage decisional process where the monop ol ist ..rst chooses whether to produce one or two qualities, in the second stage quality levels are set and in the third it chooses market price(s). ${ }^{14}$

\subsection{Consumers}

Consumers are heterogeneous in two respects. First, we assume a continuum of consumers identical in tastes but with dixerent preferences for quality $\mu$ (like in Mussa and R osen (1978)). Consumers are uniformly distributed over the interval $[\underline{\mu} ; \bar{\mu}]$, with $\underline{\mu}>0$. Second, consumers dixer in their cost of pirating the good. This assumption is introduced to ensure the production of

\footnotetext{
${ }^{12}$ We consider a situation where piracy cannot be completely eliminated by copyright protection laws.

${ }^{13} \mathrm{As}$ to the variable costs, it would be equivalent to assume a constant marginal cost of production. The absence of quality-dependent ..xed costs, besides making the analysis more tractable, helps us to isolate the impact of piracy on the incentives to dixerentiate from that generated by a costly production of quality. It is in fact a well established result that a monopolist dixerentiates its quality in the presence of quality costs (see Spence, 1975 and Mussa and Rosen, 1978).

${ }^{14} \mathrm{~T}$ his assumption on the structure of the decisional process is intended to capture the idea that the price can in practice be varied at will, while a change in the quality speci..cation of the good involves a modi..cation of the appropriate "production facilities" (Shaked and Sutton, 1982).
} 
an high-quality good in equilibrium. ${ }^{15} \mathrm{~A}$...rst group of consumers sustains a high cost $\mathrm{C}_{\mathrm{H}}$ when buying a non-original copy of the good. Consumers with cost $c_{H}$ have also high preference for quality $\mu 2[\hat{\mu} ; \bar{\mu}]$. A second group of consumers has a low cost of copying, $C_{L}$, with $C_{H}>C_{L}$. Consumers with cost $C_{L}$ have preference for quality $\mu 2[\underline{\mu} ; \hat{\mu}]$. A ssuming that a higher cost of copying is associated with higher taste for quality is rather plausible and empirically relevant. ${ }^{16}$ This is even clearer if we reinterpret consumers' heterogeneity not in terms of taste for quality, but rather in terms of income. In fact, $c_{i}$ can also be considered as the opportunity cost of going to the illegal market or spending time and resources to make a copy. Under this interpretation, richer people (with high willingness to pay for quality) usually prefer to buy the original rather than a pirated good.

We are examining a case where all individuals consume the good, eit her purchased or copied (we call this "covered market", even though not all consumers necessarily buy the good but some pirate it). ${ }^{17}$ Each consumer consumes one unit of the good. The utility of a consumer of type $\mathrm{i}=\mathrm{H} ; \mathrm{L}$ with preference for quality $\mu 2[\mu ; \mu]$ when she legally buys quality $k$ is

$$
U\left(\mu ; q_{k} ; p_{k}\right)=\mu q_{k} i \quad p_{k}
$$

whereas, when she obtains a copy is

$$
U\left(\mu ; q_{k} ; c_{i}\right)=\mu q_{k} ; \quad c_{i}
$$

\footnotetext{
${ }^{15}$ If consumers were identical in their costs of copying, $c$; and the monopolist produces two qualities at prices $p_{1}$ and $p_{2}, p_{1}<p_{2}$ the following cases are possible. i) If $c<p_{1}<p_{2}$, consumers would rather pirate both goods than purchase them. The demand for both goods is zero; ii) if $p_{1} \cdot c<p_{2}$, no consumer is willing to buy $q_{k}$, so that the latter is not produced; iii) if $p_{1}<p_{2}$. $c$, prices are so low that no consumer will pirate. The monopolist will then produce a single quality level.

It is then clear that any price strategy eliminates both piracy and the incentive for vertical dixerentiation. Therefore, only when consumers are heter ogeneous in their cost c; product dixerentiation can be pro.table.

${ }^{16} \mathrm{~W}$ e al so examined a case in which the relationship between the cost of pirating $\mathrm{c}_{\mathrm{i}}$ and the preference for quality $\mu$ is negative, i.e. consumers with $\mu 2[\mu ; \hat{\mu}]$ have a high cost of pirating $\left(c_{L}\right)$ while those with $\mu 2[\hat{\mu} ; \bar{\mu}]$ sustain a cost $C_{H}$. R esults are qual itatively similar to those presented in this article and vertical dixerentiation might still be an equilibrium strategy of the ..rm.

${ }^{17} \mathrm{~T}$ he assumption of a covered market con..guration when the monopolist is producing a single quality is actually not restrictive in the presence of piracy. In fact, it turns out that the uncovered market con..guration is less pro..table than letting all individuals consume the good. More speci..cally, when the market is not fully covered, pro..ts are $i=\frac{p}{\bar{\mu}_{i} \underline{\mu}}\left(\bar{\mu}_{i} \frac{p}{q}\right)$. No matter the price strategy adopted, it can be shown that these pro..ts are always lower than the ones obtained with a covered market con..guration.
} 
Given that we mainly refer to digital goods, we assume that the original good and its copies are identical in terms of quality. We use the expressions "pirated good" and "copy" indixerently: our de. nition of a copy includes both a copy made by somebody else and bought illegally 18 (i:e: a CD from an unauthorized vendor) and a copy made by the consumer herself ( $\mathrm{i}: \mathrm{e}: \mathrm{a}$ $C D$ copied with a CD burner). (Net) utility of the original good dixers from that of the copy because the consumer bears dixerent costs when purchasing the original good and when obtaining a copy. In the case of purchase (on the legal market), the cost is clearly the market price of the good. In the case of a copy, the cost $c_{i}$ can represent both a cost of purchasing the good on the illegal market, which also embodies a psychological cost of not having the original good, and/ or the material cost of making the copy (i:e: the price of the CD burner). This justi..es dixerent costs for dixerent consumers.

Quality $k$ is purchased rather than downloaded if and only if

$$
\mu q_{k} \text { i } p_{k}, \mu q_{k} \text { i } c_{i}
$$

which yields

$$
p_{k} \cdot c_{i}
$$

We assume that when indixerent between pirating and purchasing a good, consumers always purchase it.

\subsection{The benchmark case: no piracy}

The goal of our paper is to show that the possibility of copying may induce the monopolist to dixerentiate its product. The issue is relevant since, as the following Proposition shows, if copying is not an available option for consumers, no vertical dixerentiation would arise in equilibrium.

Proposition 1 When consumers cannot pirate the good, the pricing strategy of the monopolist entails no dixerentiation.

Proof. See A ppendix.

Given this benchmark case, in the next sections we study the impact of piracy on the pro..t-maximizing strategies of the monopolist. We ..rst analyze price and quality decisions when the monopolist produces one and two qualities respectively. We then compare the pro..ts obtained under both cases, in order to ..nd the equilibrium strategy in terms of the number and level of the qualities produced.

\footnotetext{
${ }^{18}$ In order for this interpretation to work, we should assume that on the illegal market there is a large number of identical ..rms competing à la Bertrand and where ther efore market price is equal to marginal cost.
} 


\section{The monopolist producing only one quality}

We ..rst examine the case where the monopolist produces only one quality and determine its equilibrium strategy in terms of price and quality choice.

The monopolist has three selling strategies: set such a high price that nobody buys the good $\left(\mathrm{c}_{\mathrm{L}}<\mathrm{C}_{\mathrm{H}}<\mathrm{p}\right)$, set an intermediate price that keeps only high-cost consumers in the market $\left(C_{\mathrm{L}}<\mathrm{p}<\mathrm{C}_{\mathrm{H}}\right)$, or set such a low price that both types of consumers buy $\left(\mathrm{c}_{\mathrm{C}}<\mathrm{C}_{\mathrm{H}}<\mathrm{p}\right)$. C Clearly, if $\mathrm{C}_{\mathrm{L}}<\mathrm{C}_{\mathrm{H}}<\mathrm{p}$; both the high-cost and the low-cost type prefer to pirate the good rather than purchasing it (see condition 4) and the monopolist earns zero pro..ts. We now examine the other two strategies in turn.

Lemma 2 If $p \cdot c_{L}<c_{H}$ all consumers buy the good. In equilibrium, the price is

$$
p^{a x}=C_{L}
$$

quality is

$$
q^{\alpha}=\frac{C L}{\underline{L}}
$$

whereas total pro..ts are

$$
I^{x}=C_{L}
$$

Proof. See appendix.

Lemma 3 If $c_{L}<p<c_{H}$ only high-cost consumers buy the good. In equilibrium the price is

$$
p^{x x}=C_{H}
$$

quality is

$$
q^{a x a}=\frac{C_{H}}{\hat{\mu}}
$$

whereas total pro..ts are

$$
I^{\infty}=\frac{\mu_{i} \hat{\mu}}{\mu_{i} \underline{\mu}}
$$

Proof. See appendix.

By comparing equilibrium pro.ts under the two strategies, it turns out that the optimal strategy for prices and quality depends on the degree of cost heterogeneity among consumers as described by the following proposition: 
Proposition 4 The optimal strategy entails pricing such that high-cost consumers buy and low-cost consumers pirate the good if and only if

$$
c_{L}<c_{H} \frac{\bar{\mu}_{i} \hat{\mu}}{\bar{\mu}_{i} \underline{\mu}}
$$

Otherwise, the monopolist sets a price that induces both types of consumers to buy.

Proof. See A ppendix.

The relative pro..tability of the two strategies depends on two elements: the relative size of the costs of pirating and the proportion of high-cost consumers. The higher $\mathrm{C}_{\mathrm{L}}$ with respect to $\mathrm{C}_{\mathrm{H}}$, the more it is pro.table to sell also to low-cost consumers (at price $\mathrm{C}_{\mathrm{L}}$ ). If there are many high-cost consumers it is more pro..table to set a price such that only these consumers buy $\left(p=c_{H}\right)$ rather than setting a lower price $\left(p=c_{L}\right)$ that induces also low-cost consumers to buy because the lack of pro..ts incurred in the lowcost segment of the market is more than compensated by the higher price the monopolist can charge in the high-cost segment. Conversely, if $c_{L}$ and $\mathrm{C}_{\mathrm{H}}$ are close enough and the proportion of high-cost consumers is small, it is better to set a low price such that both types of consumers purchase the good instead of letting low-cost pirate it.

It is immediate to check that $p^{\alpha}<p^{a x}$ al ways and $q^{a}<q^{a x} i C_{L}<C_{H}$ :

\section{The monopolist producing two qualities}

In this section we analyze the strategy of producing two dixerent qualities, $q_{1}<q_{2}$. We solve backwards by ..rst computing pro..ts under the two alternative strategies of allowing some piracy from low-cost consumers $\left(p_{1}<c_{L}<p_{2} \cdot c_{H}\right)$ and of eliminating piracy at all $\left(p_{1}<p_{2} \cdot c_{L}<c_{H}\right)$ :

As to the latter strategy, it reduces to the situation where copying is not an available option for consumers and the monopolist ..nds optimal not to dixerentiate (see Section 2.3).

Therefore, when considering dixerentiation, we only have to analyze the case where the monopolist chooses prices to allow piracy to exist. In this case, low-cost consumers buy quality 1 and pirate quality 2 . Notice ..rst that setting $p_{1}=C_{L}$ would imply that low-cost consumers always prefer to pirate the high-quality good rather than buying the low-quality one (given that $\mu q_{2}$ i $C_{L}>\mu q_{1}$ i $C_{L}$ ): the low-quality good would never be produced and we would be back to the one-quality case. Therefore setting $p_{1}=q$ is not an optimal strategy for the monopolist. 
Among low-cost consumers, the consumer who is indixerent between purchasing quality 1 and pirating 2 has willingness to pay $\alpha$, where $\tilde{\alpha}$ solves

$$
\mu q_{2} \text { i } C_{L}=\mu q_{1} \text { i } p_{1}
$$

and is equal to

$$
\alpha=\frac{c_{L} ; p_{1}}{q_{2} i q_{1}}
$$

The behavior of high-cost consumers is described in the following Lemma. Lemma 5 If $\hat{\mu}>\frac{\hat{\omega}}{2}$ all high-cost consumers with $\mu 2 \hat{h^{\prime}} ; \hat{\mu}$ buy quality or: If $\hat{\mu}<\frac{w}{2}$; there exjst a threshold $\mu^{ \pm} 2 \hat{\mu_{i}} ; \hat{\mu} ; \mu^{ \pm}, \frac{p_{2 i} p_{1}}{q_{k i}} ;$ such that all consumers with $\mu 2 \hat{\mu_{i}} \mu^{ \pm}$buy quality $q_{1}$ and those with $\mu 2 \mu^{ \pm} ; \mu^{\infty}$ buy quality $q_{2}$ : Proof. See appendix.

Lemma 5 implies that when the market of high-cost consumers (whose dimension is exogenously ..xed) is relatively small, it is never optimal to segment it.

We can therefore distinguish two cases

1. $\hat{\mu}>\frac{1}{2}$ so that $\mu^{ \pm}<\hat{\mu}$ : here high-cost consumers buy quality $q_{2}$ only.

2. $\hat{\mu}<\frac{1}{2}$ so that $\mu^{ \pm}>\hat{\mu}$ : here high-cost consumers buy both qualities.

We examine both cases separately, since they have dixerent implications in the choice of quality levels. In particular, as we will see, the choice of $q_{1}$ in case 1 does not infuence the pro..ts obtained in the high-cost segment as it happens instead in case 2 .

Demands for the two goods are de. ned as follows. Demand for the good of quality 1 is

$$
x_{1}={\frac{1}{\bar{\mu}_{i} \underline{\mu}}}^{3} \mu_{i} \underline{\mu}+\mu^{ \pm} i \hat{\mu}
$$

since it is bought by low-cost consumers with $\mu 2 \mu$; $; \alpha$ and by high-cost consumers with $\mu 2 \hat{\mu} ; \mu^{ \pm}$:

Similarly, demand for the good of quality 2 is

$$
x_{2}=\frac{1}{\mu i \underline{\mu}} \bar{\mu}_{i} \mu^{{ }^{\ddagger}}
$$


Finally, piracy occurs for all consumers with $\mu 2 \stackrel{h}{h} ; \hat{\mu}$ and is de..ned as

$$
x_{2 P}={\frac{1}{\mu_{i} \underline{\mu}}}^{3} \hat{\mu}_{i}{ }^{\prime}
$$

We now examinethe two casesillustrated by Lemma 5 separately, starting from the case with $\hat{\mu}>\frac{1}{2}$; where high-cost consumers buy the high quality good only.

\subsection{High-cost consumers buy quality on only}

In this case, high-cost consumers buy quality $q_{2}$ only. Low-cost consumers with $\mu<\kappa$ buy $q_{1}$ and those with $\mu>\alpha$ pirate $q_{2}$.

The equilibrium strategy for prices and quality is described by the following lemma:

Lemma 6 When $\hat{\mu}>\frac{1}{2}$, the monopolist chooses $\left(q_{1} ; q_{2}\right)$ such that the demand for good 1 is maximized and therefore piracy does not occur in equilibrium. Equilibrium prices and qualities are respectively

$$
p_{1}^{\text {diff }}=\frac{C_{L} \hat{\mu}_{i} \underline{\mu}}{2 \hat{\mu}_{i} \underline{\mu}} ; p_{2}^{\text {diff }}=C_{H}
$$

and

$$
q_{2}^{\text {diff }}=\frac{C_{H}}{\hat{\mu}} ; q_{1}^{\text {diff }}=\frac{C_{H} 2 \hat{\mu}_{i} \underline{\mu} i, c_{L} \hat{\mu}}{\hat{\mu} 2 \hat{\mu}_{i} \underline{\mu}}
$$

Equilibrium pro..ts are

$$
i^{\operatorname{diff}}=\frac{\mathrm{C}_{\mathrm{L}} \hat{\mu}_{\mathrm{i}} \underline{\mu}^{2}+\mathrm{c}_{\mathrm{H}_{3}}{ }^{3} \hat{\mu}_{\mathrm{i}} \hat{\mu}^{3}, 2 \hat{\mu}_{\mathrm{i}} \underline{\mu}}{{ }^{\mathrm{i}_{1}}{ }_{\mathrm{i}} \underline{\mu}^{\mathrm{C}^{2}} 2 \hat{\mu}_{\mathrm{i}} \underline{\mu}}
$$

Proof. See A ppendix.

It can be readily veri..ed that $q_{1}^{\text {diff }}$ is increasing in $\mathrm{C}_{\mathrm{H}}$ and decreasing in $C_{L}$, whereas $q_{2}^{\text {dif } f}$ is increasing in $C_{H}$ : $q_{1}^{\text {diff } f}$ is decreasing in $q_{L}$ because the lower the cost of pirating, the higher the quality the monopolist has to oxer to low-cost consumers to induce them to buy the good. Pro..ts in (18) are always positive.

In this dixerentiating equilibrium, the monopolist eliminates piracy not through low prices (i.e. setting $p_{1}<p_{2} \cdot C_{L}<C_{H}$ ) but through an appropriate choice of quality levels. This is done by ..xing such a high level of $q_{1}$ that all low-cost consumers decide to buy it. 


\subsection{High-cost consumers buy both qualities}

In the previous section, pro..ts from selling in the high-cost segment of the market do not depend on $q_{1}$ and this clearly infuences its equilibrium level. In particular, $q_{1}$ positively axects the demand of low-cost consumers while not having any impact on the demand of high-cost consumers (which depends only on $c_{2}$ ). When instead $\hat{\mu}<\frac{1}{2}$, i.e. the proportion of high-cost consumers is high, the impact of $q_{1}$ on pro..ts is ambiguous. In this case, high-cost consumers buy both qualities, so that an increase in $q_{1}$ decreases pro..ts in this segment through its infuence on $p_{1}$. If $p_{1}$ is low enough, high-cost consumers may in fact decide to buy $q_{1}$ rather than $q_{2}$ (more pro..table for the monopolist due to $p_{2}>p_{1}$ ). In particular, the more the two quality levels are close to each other, the lower the prices that can be set for the two goods, and therefore the lower the pro..ts that can be extracted from the high-cost segment of the market. Therefore, $q_{1}$ is not necessarily set so high that the demand for the pirated good is zero and in the equilibrium we might experience both dimerentiation and piracy.

In this case, high-cost consumers with $\mu<\mu^{ \pm}$buy quality $q_{1}$, while those with $\mu>\mu^{ \pm}$buy quality $q_{2}$ : As before, low-cost consumers with $\mu<\alpha$ buy $q_{1}$ and those with $\mu>\tilde{\mu}$ pirate $q_{2}$ :

The equilibrium strategy for prices and quality is described by the following lemma, where $f^{\prime}\left(q_{2}\right.$ i $\left.q_{1}\right)$. In what follows, the absolute levels of $q_{1}$ and $o_{\mathrm{Z}}$ are irrel evant and only their dixerence matters. ${ }^{19}$

Lemma 7 When $\hat{\mu}<\frac{1}{2}$, the monopolist always chooses either maximum or minimum dixerentiation.

1. W ith minimum dixerentiation, qualities are chosen such that their dif-

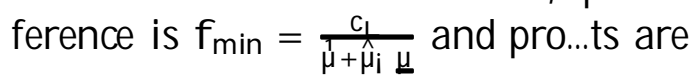

$$
i \min =\frac{c_{L} 5 \mu^{2} i \underline{3}^{4} 2 \mu_{i} \underline{\mu}_{,}^{\phi}}{4^{1} \mu_{i} \underline{\mu}^{\psi^{3}} \hat{\mu}+\hat{\mu} i \underline{\mu}}
$$

Here, $x_{1}$ is set as high as possible and no piracy arises, i.e. $\mathrm{x}_{2 \mathrm{P}}=0$.

2. W ith maximum dixerentiation, qualities are chosen such that their dif-

\footnotetext{
${ }^{19} \mathrm{~T}$ his is a typical feature of model s of vert ical dixerentiation assuming a covered market and no costs of quality improvement.
} 


$$
\begin{aligned}
& \text { ference is } f_{\max }=\frac{2 c_{\mu} i_{i}}{2 \hat{k}_{i} \hat{\mu_{i}} \downarrow} \text { and pro..ts are }
\end{aligned}
$$

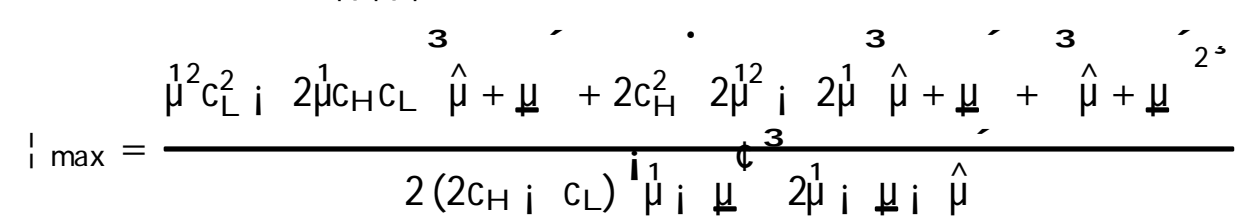

In this case, demand for piracy is

$$
x_{2 P}=\frac{2 c_{H}{ }^{3} \mu+\hat{\mu}_{i} \underline{\mu} i c_{L}{ }^{i} \mu_{i} 2 \mu^{\phi}}{2\left(2 c_{H} i c_{L}\right)}
$$

which is positive if the condition

$$
c_{L}<c_{H} \frac{2^{3} \stackrel{1}{\mu}+\hat{\mu}_{i} \underline{\mu}}{3 \underline{\mu} \mu_{i} 2 \underline{\mu}}
$$

holds.

Proof. See A ppendix.

It is interesting to notice that condition (22) in Lemma 7 implies $f_{\max }>$ $f_{\min }$ because, with $f=f_{\min } ; X_{2 P}=0$ by de. nition of $f_{\min }$ : Notice also, from (21) that $\frac{\alpha_{2 p}}{a_{1}}<0$; that is the extent of the piracy allowed by the monopolist decreases with $C_{L}$ : This happens because with a high $C_{L}$ the monopolist is able to charge a higher price for the low quality without violating the constraint $p_{1} \cdot c_{L}$ and is therefore able to produce a higher quality $q_{1}$; therefore diverting more consumers from the pirated good.

The monopolist will choose either minimum or maximum dixerentiation according to the parameters' constellation. In any case, we must make sure that two conditions are always satis..ed. Speci..cally, quality op must be sul ciently higher than $q_{1}$; so that $\alpha \cdot \hat{\mu}$, i.e. $x_{2 p}, 0$ (the demand for the pirated good is non negative). In addition, we require on to be such that its demand by low-cost consumers is non negative too (that is $\underline{\mu}<\tilde{\mu}$ ). Both conditions are met ix:

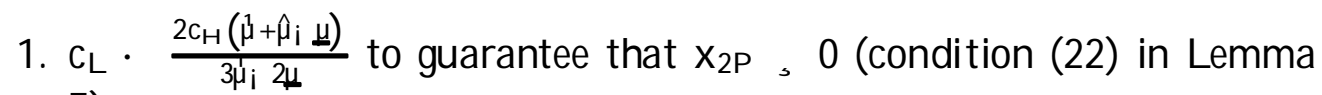
7)

2. $C_{L}>\frac{2 c_{H}\left(\hat{\mu}_{i} \hat{\mu}+\underline{\mu}\right)}{3 \hat{\mu}_{i} 2 \hat{\mu}}$ to ensure that $\alpha>\underline{\mu}$ : 
Which, given that $\frac{2 c_{H}\left(\hat{\mu}_{i} \hat{\mu}+\underline{\mu}\right)}{3^{\hat{\lambda}} \hat{\mu}_{i} \hat{\mu}}<\frac{2 c_{H}\left(\hat{\mu}+\hat{\mu_{i}} \underline{\mu}\right)}{3\left(\mu_{i} i \underline{\mu}\right.}$ always when $\hat{\mu}>2 \hat{\mu}$; can be rewritten as a unique condition

$$
\frac{2 c_{H}{ }^{3} \mu_{i} \hat{\mu}+\underline{\mu}}{3 \mu_{i} 2 \hat{\mu}}<c_{L} \cdot \frac{2 c_{H} \stackrel{3}{\mu}+\hat{\mu}_{i} \underline{\mu}}{3 \underline{\mu} i 2 \underline{\mu}}
$$

As we will need to refer to condition (23) several times in what follows, we de..ne

$$
\begin{aligned}
& \underline{c}_{L}=\frac{2 c_{H} \stackrel{1}{\mu}_{i} \hat{\mu}+\underline{\mu}}{3 \mu \mu_{i} 2 \hat{\mu}}, \\
& \varepsilon_{L}=\frac{2 c_{H} \hat{\mu}+\hat{\mu}_{i} \underline{\mu}}{3 \hat{\mu} i 2 \underline{\mu}}
\end{aligned}
$$

The following proposition establishes the conditions such that maximum dixerentiation yields higher pro..ts than minimum dixerentiation:

Proposition 8 When $\hat{\mu}<\frac{\mu}{2}$ and the monopolist dixerentiates its product, there exists a value for $\hat{\mu}$;

$$
\hat{\mu_{1}}=\frac{\mu^{2}}{4 \stackrel{\mu}{\mu} \underline{i} \underline{\underline{\mu}}}
$$

such that:

1. If $\hat{\mu}<\hat{\mu}_{1}$ maximum dixerentiation yields overall higher pro..ts than minimum dixerentiation.

2. If $\hat{\mu}>\hat{\mu}_{1}$ there exists a value for $c_{L} ; \epsilon_{1}$; such that minimum dixerentiation yields higher pro..ts for all $\epsilon_{1}<c_{L}<\xi_{L}$ and maximum dixerentiation yields higher pro.ts for $\underline{\mathrm{C}_{L}}<\mathrm{C}_{\mathrm{L}}<\mathrm{\epsilon}_{\mathrm{L}}$ :

Proof. See A ppendix.

The intuition for this result can be given as follows. When $\hat{\mu}$ is low (the situation depicted in Figure 1 ) the high-cost segment of the market, consisting of customers with $\mu 2 \hat{\mu} ; \hat{\mu}$; is large. Therefore, the monopolist would rather produce highly dixerentiated quality levels to be sold at high prices (notice that $\frac{\frac{a q}{\Phi}}{\Phi}>0$; that is prices depend positively on the dixerence in quality levels) than selling at lower prices qualities that are close substitutes. 
In other words, pro..ts from selling at high prices ${ }^{20}$ on the high segment of the markf mpre than compensate the loss of customers due to piracy in the segment $\tilde{\mu} ; \hat{\mu}$ :

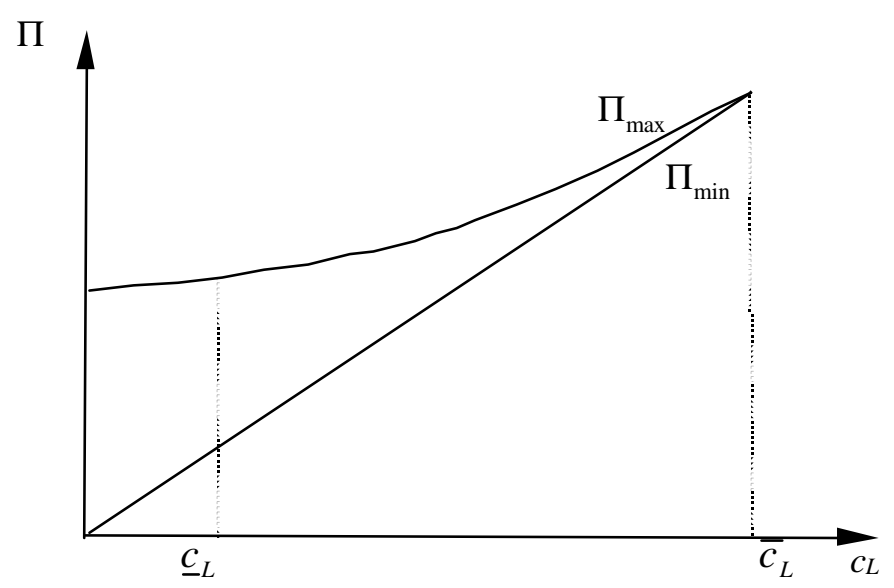

Figure 1

Conversely, when $\hat{\mu}$ is high (the situation depicted in Figure 2), the highquality segment of the market is small and the pro..t accruing from it little. The monopolist prefers maximum dixerentiation only when $c_{L}$ is very low. This happens because, when $C_{L}$ is close to zero, $1 \mathrm{~min}$ is close to zero too (both prices and the dixerence between qualities, $f_{\min }$ tend to zero) whereas with maximum dixerentiation prices are both high and pro..ts from the high segment of the market high too notwithstanding the high number of low-cost consumers pirating the good. ${ }^{21}$

\footnotetext{
${ }^{20} \mathrm{~T}$ he price for the high quality when the monopolist applies maximum dixerentiation, $\mathrm{p}_{2}=\mathrm{c}_{\mathrm{H}}$; is higher than the price for the high quality with minimum dixerentiation. Therefore, pro..ts from selling to the high-cost segment of the market (consumers with $\mu 2 \frac{\vec{\mu}}{2} ; \vec{\mu}$ ) are higher with maximum dixerentiation. The same applies to $p_{1}$ :

${ }^{21}$ It is possible to show that demand for the low-quality good from the low-cost consumers (demand de..ned as $x_{1}^{L}=\frac{a_{i}-\underline{\mu}}{\mu_{i} \underline{\mu}}$ ) is increasing in $C_{L}$ :
} 


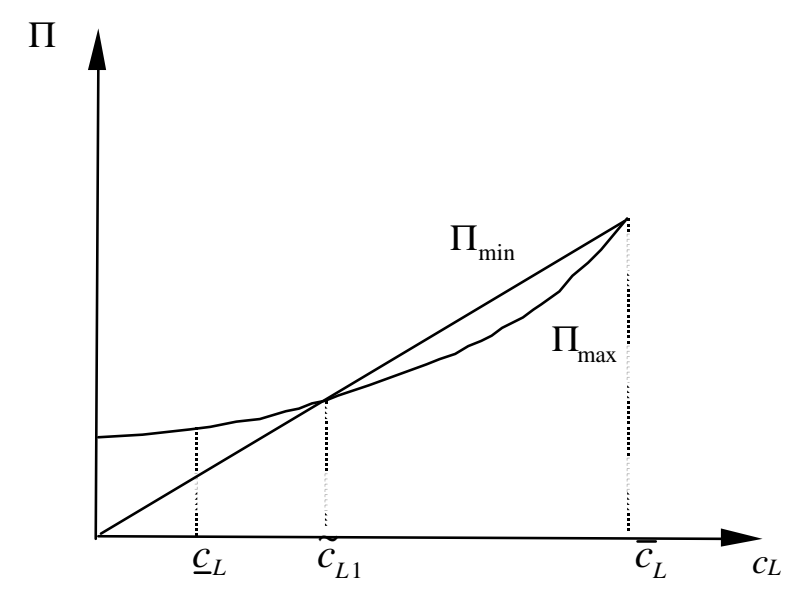

Figure 2

Next section compares all strategies available to the monopolist, with the aim to establish when product dixerentiation is an equilibrium strategy and the conditions under which piracy occurs in the market.

\section{One or two qualities?}

In the ..rst stage, the monopolist chooses whether to produce one or two qualities. In order to make this decision, it compares pro..ts from each available alternative. The main ..nding is that piracy may induce the monopolist to produce dixerent qual ities even when there are no production costs. In fact, the existence of piracy itself may justify the introduction of lower qualities, in the attempt to move away consumers with lower willingness to pay from the illegal market, where copies of the high quality good are exchanged, and let them buy the low quality instead. When the proportion of high-cost consumers is low, in equilibrium the monopolist may produce a low quality that is high enough to be appealing to all consumers: this will eliminate demand for copies and hence piracy. P iracy is however observed in many markets. In our model, the presence of piracy may arise in a dixerentiating equilibrium when the proportion of high-cost consumers is high.

\subsection{High-cost consumers buy quality or only}

When $\hat{\mu}>\frac{1}{2}$, in equilibrium the monopolist produces two quality levels only if this yields higher pro.ts than producing one quality only (by setting prices such that piracy is either eliminated or tolerated). 
The following proposition indicates the conditions under which producing two qual ities is the equilibrium strategy.

Proposition 9 In equilibrium, the monopolist chooses to produce two quality

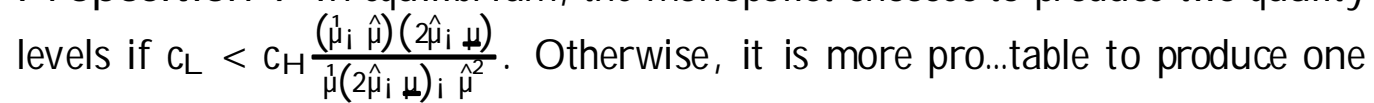
quality. In both cases, prices and quality levels are set such that no piracy arises.

Proof. See A ppendix.

The intuition for this result can be given by Figure 3 below. The pro..t when the monopolist dixerentiates, i diff; is always above ; (the pro..t with one quality only and piracy) but for the case where $C_{L}=0$. This can be seen immediately by rewriting $i$ diff in expression (18) as follows

$$
i^{\text {diff }}=c_{H} \frac{\mu_{i} \hat{\mu}}{\mu_{i} \underline{\mu}}+c_{L} \frac{\hat{\mu}_{i} \underline{\mu}}{2 \hat{\mu}_{i} \underline{\underline{\mu}}}=i^{\infty}+c_{L} \frac{\hat{\mu}_{i} \underline{\mu}}{2 \hat{\mu}_{i} \underline{\underline{\mu}}}
$$

Producing one quality while allowing some piracy is never an equilibrium strategy because the monopolist could always pro.tably introduce another (lower) quality which would attract at least some of the low-cost consumers that would have otherwise pirated the good. For very high levels of $C_{L}$ (close to $\mathrm{C}_{\mathrm{H}}$ ) the monopolist prefers to produce one qual ity because a high price $p$ - $c_{L}$ can be set and it is then pro..table to sell the same (high) quality to the whole market. If $\mathrm{C}_{\mathrm{L}}$ is low and $\mathrm{C}_{\mathrm{H}}$ is much higher than $\mathrm{C}_{\mathrm{L}}$, a strategy such that $p_{1}<c_{L}<p_{2} \cdot C_{H}$ is more pro..table, where the monopolist sells to the high-cost segment of the market at a very high price and captures the low-cost segment by selling a low-price, low-quality good.22

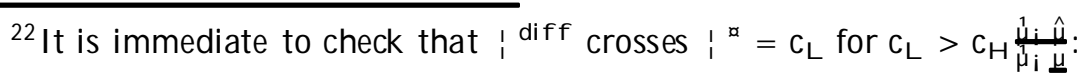




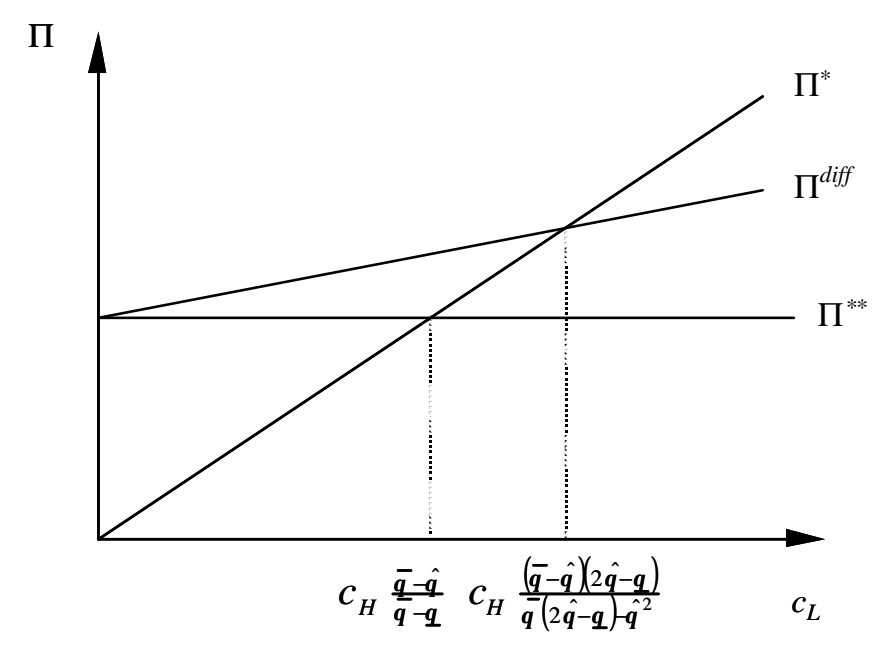

Figure 3

\subsection{High-cost consumers buy both qualities}

We now proceed by showing the existence of an equilibrium with maximum dixerentiation (as well as equilibria with one quality only).

We obtain the following results.

Proposition 10 There exist one value for $\mathrm{C}_{\mathrm{L}} ; \mathrm{C}_{\mathrm{L}}^{\text {pir }}$; such that

1. If $\hat{\mu}<\hat{\mu}_{1}$ the following cases arise:

(a) if $\hat{\mu}>{ }^{i} 3+{ }^{p}{ }_{5}^{\Phi} \underline{\mu}$ then: if $\hat{\mu}_{2}<\hat{\mu}<\hat{\mu}_{1}$ the monopolist chooses maximum dixerentiation for all $\mathrm{C}_{\mathrm{L}}^{\mathrm{pir}}<\mathrm{C}_{\mathrm{L}}<\mathrm{E}_{\mathrm{L}}$ and produces one quality allowing piracy if $c_{L}<c_{L}^{\mathrm{pir}}$; if $\hat{\mu}<\hat{\mu}_{2}<\hat{\mu}_{1}$ then the monopolist always produces one quality allowing piracy;

(b) if ${ }_{\mu}^{1}<{ }^{i} 3+{ }^{P} \overline{5} \underline{\mu}$ the monopolist always produces one quality allowing piracy.

2. If $\hat{\mu}>\hat{\mu}_{1}$ the monopolist produces one quality, either by letting low-cost consumers pirate the good (if $\mathrm{C}_{\mathrm{L}}<\mathrm{C}_{\mathrm{H}} \frac{\mathrm{p}_{\mathrm{j}} \hat{\mu}}{\mu_{\mathrm{i}} \underline{\underline{\mu}}}$ ) or by setting a price that induces both types of consumers to buy (if $c_{L}>c_{H} \frac{\mu_{i} \hat{\mu}}{\mu_{i} \underline{\underline{\mu}}}$ ).

Proof. See A ppendix.

The intuition for the results in Proposition 10 can be given as follows. From Proposition 8 we know that pro.ts are higher with maximum rather 
than minimum dixerentiation when $\hat{\mu}<\hat{\mu}_{1}$ : However, it can also be shown that in this case minimum dixerentiation is better than selling one quality to the whole market: Therefore it follows that maximum dixerentiation is preferred to selling a single quality to the whole market. This latter result follows from the much higher pro..ts the monomolist can extract from the high-cost, high-quality segment of the market $\frac{\omega}{2} ; \hat{\mu} \quad$ (which now is large), where $q_{2}$ can be sold at $p_{2}=c_{H}$ instead of $p=c_{L}$ :

The relevant comparison when $\hat{\mu}<\hat{\mu}_{1}$ is therefore between $i \max$ and $;$ the curve and the straight line parallel to the $x_{i}$ axis in Figure 4 below. It is then immediate to check that $\quad>$ max for all $\underline{c}_{L}<c_{L}<c_{L}^{\text {pir }}$ : Then, for Iow $C_{L}$; selling one quality to the high-cost segment of the market is a better strategy than selling two qual ities to that segment of the market, while selling the lower quality to a share $\frac{\mu_{i}}{\mu_{i}}$ of the low-cost segment and letting all ot her consumers pirate $\mathrm{q}_{2}$ : When $\mathrm{c}_{\mathrm{L}}$ is low, thepextra pro..t the monopolist makes by selling $q_{1}$ to the consumers with $\mu 2 \underline{\text {; }} ; \alpha$ is very low (recall that $p_{1}$ is positively related to $C_{L}$ ). The loss of pro..ts from selling $q_{2}$ only to a share $\frac{\mu_{i} \frac{1}{2}}{\mu_{i}}$ of the market and $q_{1}$ at $p_{1}$ to the remaining share is instead quite substantiat. That explains why $I_{i}>$ max for low $C_{L}$ whereas the order is reversed for suc ciently high values of $C_{L}$ :

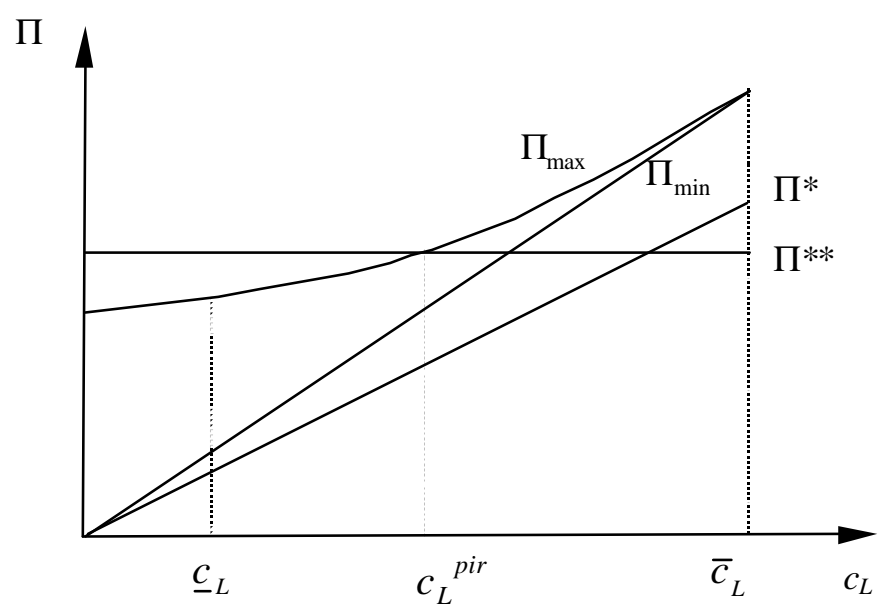

Figure 4

A less interesting case arises when ${ }_{0}$ consumers are enough homogeneous in their taste for quality $\left({ }_{\mu}^{1}<3+{ }^{3} \underline{\mu}\right)$. In this case the monopolist always ..nds more pro..table to sell a single quality to high-cost consumers only (whose proportion is relatively high given that $\hat{\mu}<\hat{\mu}_{1}$ ) rather than 
dixerentiating its product.

When $\hat{\mu}>\hat{\mu}_{1}$ and the high-cost segment of the market smaller than in the ..rst part of P roposition 10, selling one qual ity to the whole market is overall better than minimum dixerentiation. The relevant comparison therefore is between $|\max ;|^{a}$ and ${ }^{x}$ : However, it turns out that in the relevant range of the parameters, maximum dixerentiation is al ways dominated by thestrategy of selling a single quality to the whole market. Maximum dixerentiation yields lower pro..ts that selling one quality t $\phi_{\text {, }}$ the whole market because the loss from piracy from consumers with $\mu 2 \tilde{\mu}_{i} \hat{\mu}$ is not compensated by the higher pro..ts from selling a higher quality at $p_{2}=C_{H}$ in the segment $\frac{\omega}{2} ; \mu$ of the market, as such segment is too small. The relevant comparison is therefore between the strategy of selling a single quality to the whole market and the strategy of letting low-cost consumers pirate the good, as in Proposition 4. This case is depicted in Figure 5 below.

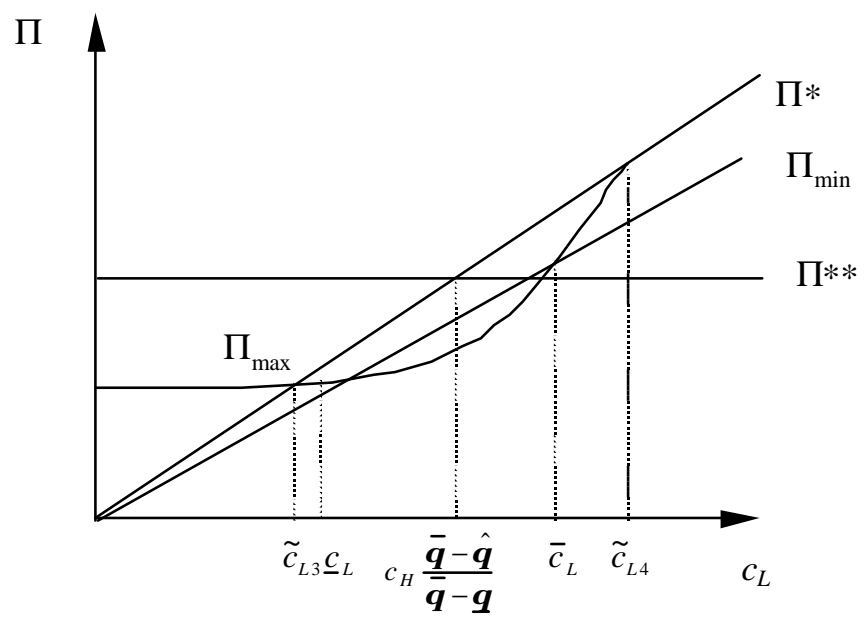

Figure 5

\section{Conclusions}

We have analyzed the choice of quality levels by a monopolist in a market where piracy exists. The main idea is that the introduction of a lower quality may be a device through which the monopolist manages to capture some consumers that would otherwise prefer to pirate the good. We have shown that there are ranges of the parameters for which the monopolist prefers to 
produce two qualities rather than one quality only. The relative pro..tability of these two strategies essentially depends on the degree of consumers' heterogeneity in the cost of pirating and on the relative proportion of the two types of consumers (those with low cost of pirating and those with high cost of pirating). In particular, we have showed that, when the proportion of high-cost consumers is low and dixerentiation is the optimal strategy, the quality level chosen for the low-quality good is such that piracy is completely eliminated in equilibrium.

When instead the proportion of high-cost consumers is high, there is an equilibrium with maximum dixerentiation where the monopolist admits some piracy. This happens because, since in this case the monop olist ..nds pro..table to sell both qualities to the high-cost segment of the market, the demand of the high-quality good depends positively on $p_{1}$. In particular, the more the two quality levels are close to each others, the lower the prices that can be set for the two goods, and therefore the lower the pro.ts that can be extracted from the high-cost segment of the market. Therefore, the low-quality is not necessarily set so high that the demand for the pirated good is zero and in the equilibrium we might experience both dixerentiation and piracy.

An interesting extension would be to evaluate the impact of more restrictive copyright laws on equilibrium prices and qualities in a market where consumers can pirate the good. We can do so by incorporating in the cost of copying the expected ..ne that the consumer has to pay if caught in possession of the illegal good and study how the monopolist's strategies would respond to an increase in the expected ..ne. We expect that an increased copyright protection, by making pirating more costly and therefore less viable for consumers, would reduce the monopolist's incentive to produce a second (lower) quality in order to reduce piracy. The degree of copyright enforcement could also be seen as an endogenous choice of the monopolist by appropriately specifying its objective function.

\section{A A ppendix: Proofs}

Proof of Proposition 1 When two quality levels are produced in a "covered market" con..guration, the demands for quality 1 and quality 2 are

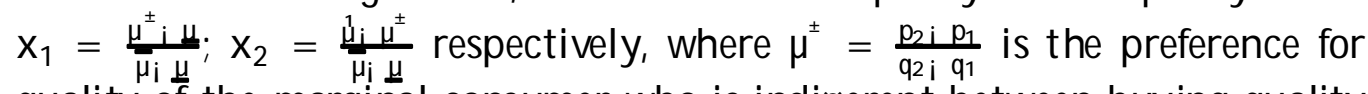
quality of the marginal consumer who is indixerent between buying quality 1 and quality 2. Pro.ts are

$$
i\left(p_{1} ; p_{2}\right)=\frac{1}{\bar{\mu}_{i} \underline{\mu}} p_{1} \frac{p_{2} i p_{1}}{q_{2} i q_{1}} i \underline{\text { q }}+p_{2} \bar{\mu}_{i} \frac{p_{2} i p_{1}}{q_{2} i q_{1}}{ }^{\text {I }} \text {. }
$$


Following the three stage decisional process described above, the monop ol ist will ..rst de.ne its optimal price strategy. Note however that $x_{1}, 0$ implies $\frac{\mathrm{G}}{\Phi_{1}}>0,8 p_{2}>0$. The monopolist would then ...nd optimal to set $p_{1}$ as high as possible, i.e such that $x_{1}=0$. This implies $p_{1}=p_{2} i \underline{\mu}\left(q_{2} ; q_{1}\right)$ and $x_{2}=1$. Therefore, in the quality stage the monopolist would choose to produce a single quality.

Proof of Lemma 2 When $p \cdot c_{L}<c_{H}$; pro..ts are $:(p)=p$ since at this price all consumers buy the good. The monopolist will set the highest possible price subject to the constraint that the consumer with the lowest willingness to pay $(\underline{\mu})$ has non-negative utility, that is

$$
p=\underline{\mu q}
$$

Total pro..ts are therefore increasing in q: The highest $q$ the monopolist can produce is the one such that $p^{\alpha}=C_{L}$ :

$$
q^{\alpha}=\frac{p}{\underline{p}}=\frac{C_{L}}{\underline{\mu}}
$$

Equilibrium pro..ts are

$$
i^{x}=C_{L}
$$

Proof of Lemma 3 When $c_{L}<p \cdot c_{H}$; high-cost consumers buy the good and low-cost ones pirate it. Demand is therefore given by the high-cost fraction of consumers

$$
x=\frac{1}{\mu_{i} \underline{\mu}}\left[\bar{\mu}_{i} \hat{\mu}\right]
$$

and pro..ts are

$$
i(p)=\frac{p}{\mu_{i} \underline{\mu}}\left[\bar{\mu}_{i} \hat{\mu}\right]
$$

Since pro..ts are increasing in $p$, the monopolist will choose the highest price such that the marginal buyer (the one with $\mu=\hat{\mu}$ ) has zero surplus, i.e. $p=\hat{\mu q}$. Plugging this into (32), we get

$$
i(q)=\hat{\mu} q \frac{\bar{\mu}_{i} \hat{\mu}}{\bar{\mu}_{i} \underline{\mu}}
$$

Pro..ts are increasing in the quality of the good and the monopolist will set $q$ as high as possible given the constraint that $p \cdot C_{H}$, i.e. $q \cdot \frac{C_{H}}{\mu}$. Hence, 
the equilibrium price and quality are

$$
\begin{aligned}
& p^{\alpha a x}=C_{H} \\
& q^{\alpha x}=\frac{C_{H}}{\hat{\mu}}
\end{aligned}
$$

The equilibrium pro.ts are

$$
=c_{H} \frac{\bar{\mu}_{i} \hat{\mu}}{\bar{\mu}_{i} \underline{\mu}}
$$

Proof of Proposition 4 The optimal strategy for the monop ol ist is determined by comparing the equilibrium pro..ts under the three strategies. Since the ..rst strategy, i.e setting such a high price that nobody purchases the good, leads to zero pro..ts and is never optimal, the relevant strategies are only the second and the third ones. It turns out that $\left.\right|^{\infty}>(i$ :e: it is better to set a price that induces both types of consumers to buy $\left(p=c_{L}\right)$ if and only if

$$
c_{L}>c_{H} \frac{\bar{\mu}_{\mathrm{i}} \hat{\mu}}{\bar{\mu}_{\mathrm{i}} \underline{\mu}}
$$

P roof of Lemma 5 High-cost consumers can either buy 1 or buy 2, and they buy 1 in

$$
\mu<\mu^{ \pm}, \frac{p_{2} \text { i } p_{1}}{q_{2} \text { i } q_{1}}
$$

Hence, demand for the good of quality 1 is

$$
x_{1}={\frac{1}{\bar{\mu}}{ }^{3} \underline{\mu}}^{3} \tilde{\mu}_{i} \underline{\mu}+\mu^{ \pm} \mathrm{i} \hat{\mu}
$$

Similarly, demand for the good of quality 2 is

$$
x_{2}=\frac{1}{\mu_{i} \underline{\mu}} i_{\bar{\mu}} \mu^{ \pm} \Phi
$$

The monopolist's pro..ts thus are

$$
i\left(p_{1} ; p_{2}\right)=\frac{1}{\bar{\mu} i \underline{\mu}} p_{1} \frac{c_{L}+p_{2} i 2 p_{1}}{q_{2} i q_{1}} i \underline{\mu} i \hat{\mu}+p_{2} \bar{\mu}_{i} \frac{p_{2} i p_{1}}{q_{2} i q_{1}} \text { ף, }
$$

The pro..t function is globally concave in $\left(p_{1} ; p_{2}\right)$ and the ..rst-order condition $\frac{@(:)}{q_{2}}=0$ gives $p_{2}=p_{1}+\frac{\bar{u}}{2}\left(q_{2}\right.$ i $\left.q_{1}\right)$, which implies $\mu^{ \pm}=\frac{\bar{u}}{2}$. Hence, high cost consumers buy the low quality good ia $\hat{\mu}<\frac{\bar{\mu}}{2}$ : 
Proof of Lemma 6 Given the demands in expression (13), (14), the monopolist's pro.ts are

$$
i\left(p_{1} ; p_{2}\right)=\frac{1}{\mu_{i} \underline{\mu}} p_{1} \frac{C_{L} i p_{1}}{q_{2} i q_{1}} i \stackrel{\text { व }}{\mu}+p_{2} \stackrel{3}{\mu}^{\prime} \hat{\mu}^{\prime},
$$

P ro.ts are al ways increasing in $p_{2}$. The monopolist ..xes the highest $p_{2}$ that makes all high-cost consumers purchase the good, i.e. such that the last consumer purchasing quality 2 has zero surplus. Hence $p_{2}=\hat{\mu} q_{2}$.

From the ..rst order condition on $p_{1}$

$$
p_{1}=\frac{q_{L} ; \underline{\mu}\left(q_{2} ; q_{1}\right)}{2}
$$

Notice that $p_{1}>0$ if and only if $c_{L}>\underline{\mu}\left(q_{2} ; q_{1}\right)$ : The second-order condition is satis.ed since $\frac{Q_{1}^{2}}{\Phi_{1}^{2}}=i 2$ : We now solve backward and obtain the optimal quality levels. Substituting $p_{1}$ and $p_{2}$ into (41), the monopolist's pro..t becomes

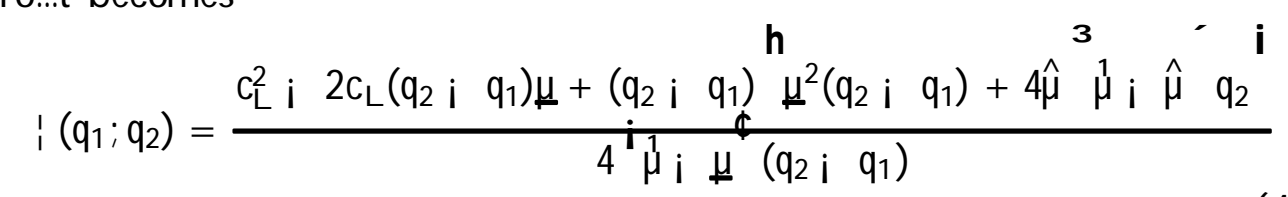

Notice that $\frac{\Phi(:)}{\varrho_{1}}=\frac{c_{1}^{2} i\left(q_{2} i q_{1}\right)^{2} \underline{\underline{\mu}}^{2}}{4\left(\hat{p}_{i} \underline{\underline{u}}\right)\left(\left(_{2} i q_{1}\right)^{2}\right.}>0$ whenever $p_{1}>0$. The monopolist thus sets $q_{1}$ as high as possible. This is done by choosing it such that $x_{1}$ is maximized and piracy disappears, i.e. $x_{2 p}=0$. Hence $q_{1}$ is set so that $\alpha=\hat{\mu}$ :

$$
q_{1}^{\text {diff }}=q_{2} i \frac{C_{L}}{2 \hat{\mu} i \underline{\mu}}
$$

This means that the last consumer pirating the good (i.e. $\tilde{q}$ ) is made indifferent between buying and pirating good 2 . Subject to the constraint that $q_{1}=q_{1}^{\text {diff }}$ pro..ts are now equal to:

$$
i\left(q_{2}\right)=\frac{c_{L} \hat{\mu}_{i} \underline{\mu}^{2}+q_{2}{ }^{3} \mu_{i} \hat{\mu} \hat{\mu}^{3} 2 \hat{\mu}_{i} \underline{\mu}}{{ }^{i} \mu_{i} \underline{\mu}^{4} 2 \hat{\mu}_{i} \underline{\mu}}
$$

A gain, $\frac{Q_{(}\left(q_{2}\right)}{\varrho_{2}}>0$ and $q_{2}$ is set at its highest possible level. Since prices are constrained by the condition $p_{1}<c_{L}<p_{2} \cdot c_{H}$ and since $p_{2}=\hat{\mu} q_{2}$; the maximum $q_{2}$ has to satisfy $\hat{\mu} q_{2}=c_{H}$ : Thus the equilibrium level of $q_{2}$ is

$$
Q_{2}^{\text {diff }}=\frac{C_{H}}{\hat{\mu}}
$$


Substituting (46) into (44), $q_{1}^{\text {diff }}$ is equal to,

$$
q_{L}^{\text {diff }}=\frac{c_{H} 2 \hat{\mu}_{i} \underline{\mu} i, c_{L} \hat{\mu}}{\hat{\mu} 2 \hat{\mu}_{i} \underline{\mu}}
$$

Further substitutions of ( $q_{1}^{\text {diff }} ; q_{2}^{\text {diff }}$ ) into (42) $p_{2}$ and (45) yields $p_{1}^{\text {dif } f}, p_{2}^{\text {dif } f}$, I diff as written in the lemma's statement. It is immediate to check that $p_{1}^{\text {diff }}<q_{L}<p_{2}^{\text {diff }}$ and $q_{2}^{\text {diff }}>q_{1}^{\text {diff }}>0$ (As $\left.q_{2}^{\text {dif } f} i q_{1}^{\text {diff }}=\frac{a}{2 \widehat{\mu i} \psi}>0\right)$ as required. Also, pro.ts are positive for any value of the parameters.

Proof of Lemma 7 Demand for quality $q_{2}$ is now

$$
x_{2}=\frac{1}{\mu_{i} \underline{\mu}} \stackrel{f_{1}}{\mu i} \mu^{ \pm x}
$$

while demand for quality $q_{1}$ is

$$
x_{1}=\frac{1}{\mu_{i} \underline{\mu}} \mu^{3} \mu^{ \pm} \hat{\mu}+{ }^{3} \tilde{\mu}_{i} \underline{\mu}
$$

The demand for the pirated good of quality 2 is again

$$
x_{2 P}=\frac{1}{\mu i^{\mu}}\left[\hat{\mu}_{i} \quad \mu\right]
$$

The monopolist's pro.ts thus are

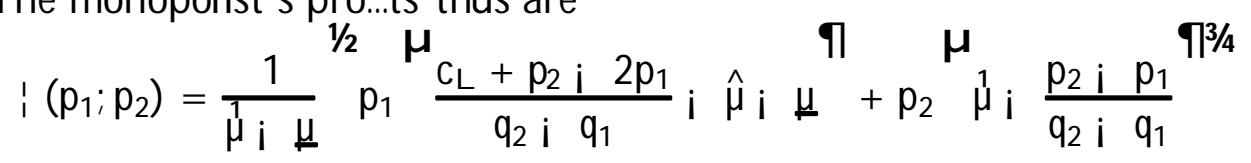

The ..rst order conditions with respect to $p_{1}$ and $p_{2}$ are respectively

$$
\begin{aligned}
& \frac{\Theta(:)}{\mathbb{Q}_{1}}=\frac{c_{L}+2\left(p_{2} i 2 p_{1}\right) i^{3} \hat{\mu}_{i} \underline{\mu}\left(q_{2} i q_{1}\right)}{\mu_{i} \underline{\varphi}\left(q_{2} i q_{1}\right)}=0
\end{aligned}
$$

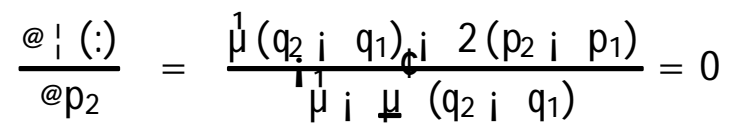

It is immediate to check that the second order conditions are satis..ed. Solving for the two prices we obtain

$$
\begin{aligned}
& p_{1}=\frac{c_{L}+f^{3}{ }^{1} \mu_{i} \hat{\mu}_{i} \underline{\mu}}{32}, \\
& p_{2}=\frac{c_{L}+f 2 \mu_{i} \hat{\mu}_{i} \underline{\mu}}{2}
\end{aligned}
$$


In the quality stage, the demands for $q_{1}$ and $q_{2}$ by high-cost consumers are $\frac{\bar{\mu}_{i} 2 \hat{\mu}}{2\left(\hat{\mu}_{i} \underline{\mu}\right)}$ and $\frac{\bar{\mu}}{2\left(\hat{\mu}_{i} \underline{\mu}\right)}$ respectively. Notice that they are independent of $f$. Pro..ts can be rewritten as

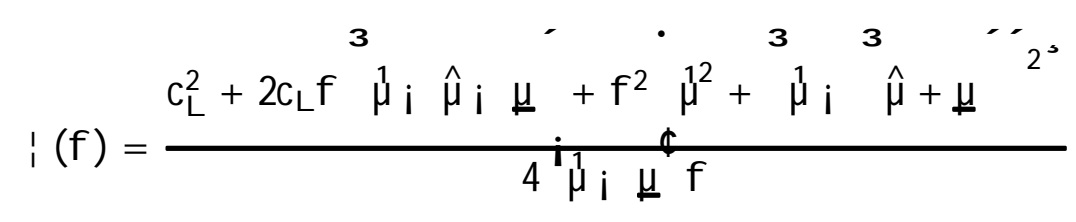

It can be noticed that pro..ts depend exclusively on $f$ and that

$$
\begin{aligned}
& \frac{d_{i}(f)}{d f}=\frac{1}{4} \frac{f^{f^{2}\left(\bar{\mu}_{i} \hat{\mu}\right)^{2}+\left(\bar{\mu}_{i} \underline{\underline{\mu}}\right)^{2}+2 \hat{\mu} \underline{\mu} i q_{L}^{2}}}{\bar{\mu}_{i} \underline{\underline{\mu}} f^{2}} \\
& \frac{d^{2} !(f)}{d^{2}}=\frac{c_{L}^{2}}{2^{i} \mu \frac{1}{\mu} \mu_{f} f^{3}}>0
\end{aligned}
$$

The pro.t. function is convex and can be either decreasing or $U$-shaped in $f$ depending on the value of the parameters. A ny critical point is therefore a minimum and optimal solutions for $f$ are to be found in the extremes, with the monopolist choosing either maximum or minimum dixerentiation for the quality levels.

Minimum Diqerentiation The pro..t maximizing choice of the monopolist will be minimum dixerentiation ( $f$ low) either when ! ( $f$ ) is decreasing or when $;$ ( $f$ ) is $U$-shaped and pro.ts from setting qualities as close as possi ble are higher than pro..ts with maximum dixerentiation. We must have that the demand for piracy is non-negative. According to (15), $x_{2 P}, 0$ ix $\hat{\mu}_{\text {i }} \tilde{\mu}, 0$; and after substituting $p_{1}$ from (53) into (15), the smallest value for $f=o_{2}$ i $q_{1}$ (that makes $\tilde{\mu}=\hat{\mu}$ ) is

$$
f_{\min }=\frac{C_{L}}{\hat{\mu}+\hat{\mu}_{i} \underline{\mu}}
$$

Since $f_{\min }>0 ; q_{2}>q_{1}$ and, from (55), total pro.ts are

$$
i \min =\frac{q 5 \mu^{12}{ }_{3}^{4} \mu^{i} 2 \mu_{i} \underline{\mu}^{q^{i}}}{4^{i} \mu_{i} \underline{\mu}^{4} \mu+\hat{\mu}_{i} \underline{\mu}}
$$

which are always positive. 
Maximum Dixerentiation $W$ ith maximum dixerentiation the monopolist sets quality $q_{2}$ at the highest possible level, whereas quality $q_{1}$ is set as low as possible. Given that $p_{2}$ in (54) is increasing in $q_{2}$; quality $q_{2}$ is set so that $p_{2}=c_{H}$ : As to $q_{1}$, it is chosen such that the utility of the consumer with the lowest willingness to pay is zero, i.e.

$$
\left\lfloor\mathrm{q}_{1} ; \quad \mathrm{p}_{1}=0\right.
$$

Substituting $p_{1}$ from (53) into (58) and setting $p_{2}$ from (54) equal to $C_{H}$, we then have a system whose solution yields the levels $q_{1}^{\max }$ and $q_{2}^{\max }$ chosen with maximum dixerentiation

$$
\begin{aligned}
& \underline{\mu} q_{1} i \frac{c_{L}+\left(q_{2} i q_{1}\right)^{3} \mu_{i} \hat{\mu}_{i} \underline{\mu}}{2_{3}}=0 \\
& q_{H} i \frac{c_{L}+\left(q_{2} i q_{1}\right) 2 \mu_{i} \hat{\mu}_{i} \underline{\mu}}{2}=0
\end{aligned}
$$

The solution is

$$
\begin{aligned}
& q_{1}^{\max }=\frac{\mu_{c_{L}}+2 c_{3}{ }^{3} \mu^{3} \hat{\mu}_{i}, \underline{\mu}}{2 \underline{\mu} 2 \mu_{i} \hat{\mu}_{i} \underline{\mu}_{3}}
\end{aligned}
$$

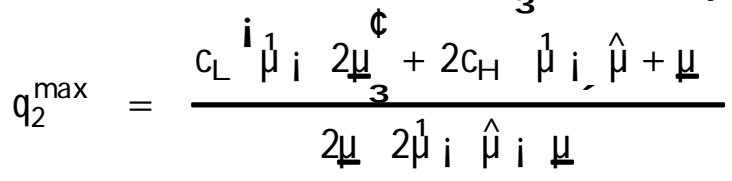

and

$$
f_{\max }=\frac{2 C_{H} i C_{L}}{2 \mu \hat{\mu}_{i} \underline{\mu}}>0
$$

Prices are

$$
\begin{aligned}
& p_{1}=\frac{c_{L} \mu_{3}+2 c_{H}{ }^{3} \mu_{i} \hat{\mu}_{i} \underline{\mu}}{22 \mu_{i} \hat{\mu}_{i} \underline{\mu}} \\
& p_{2}=c_{H}
\end{aligned}
$$

From (55), total pro..ts are

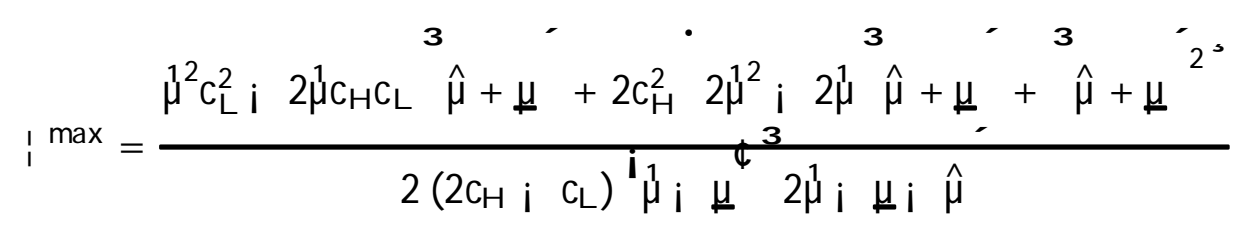


When the monopolists chooses maximum dixerentiation, from (15), demand for the pirated good is

$$
x_{2 P}=\frac{2 C_{H}{ }^{3} \hat{\mu}+\hat{\mu} i \underline{\mu} i C_{L}{ }^{i} 3 \mu_{i} 2 \underline{\underline{\mu}}}{2\left(2 C_{H} i q_{L}\right)}
$$

which is positive when $C_{L}<C_{H} \frac{2\left(\hat{\mu}+\hat{\mu}_{i} \underline{\mu}\right)}{3\left(\mu_{i} 2 \underline{L}\right.}$.

Proof of Proposition $8 \quad$ max is an increasing and convex function in $\mathrm{C}_{\mathrm{L}}$ since prices and demand functions for both goods are always non-decreasing in $C_{L}:$ The second derivative of $I$ max is

$$
\frac{d^{2} ! \max }{d c_{L}^{2}}=\frac{2 c_{H}^{2} 2 \mu_{i}^{1} \hat{\mu}_{i} \underline{\mu}}{\frac{\Phi}{\mu}+\underline{4}\left(2 c_{H} i c_{L}\right)^{3}}
$$

which is al ways positive given our assumptions on the parameters.

I min is always increasing and linear in $C_{L}$; as it is immediate to check from (57) and $\mid \min$ cuts $\mid \max$ twice for all values of the parameters. Solving

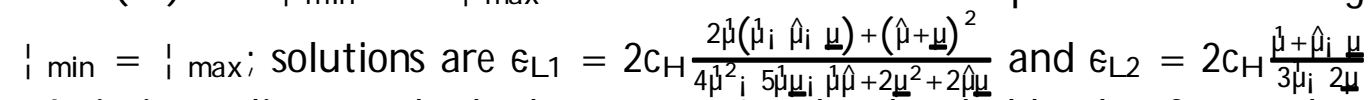
: It is immediate to check that $\epsilon_{2}=\varepsilon_{L}$; the threshold value for $C_{L}$ that guarantees that piracy is non-negative. Moreover, $\epsilon_{1}>\underline{c}_{L}$ always.

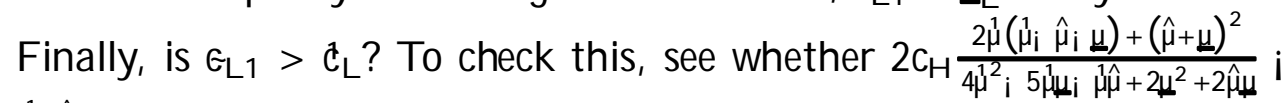
$2 c_{H} \frac{\hat{\mu}+\hat{\mu}_{i} \underline{\mu}}{3 \mu_{i} 2 \mu}>0$; that is

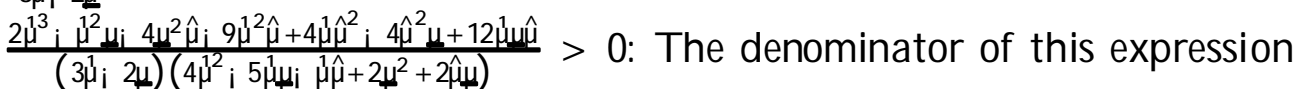

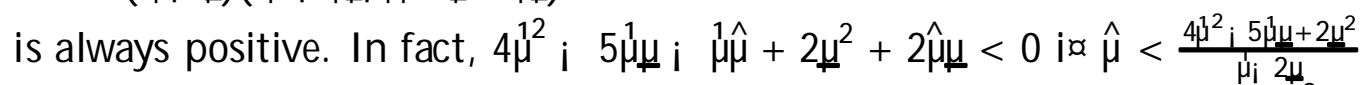
which is always true as $2 \hat{\mu}>\hat{\mu}$ :We must now check the numerator $2 \mu^{3} \mathbf{i}$

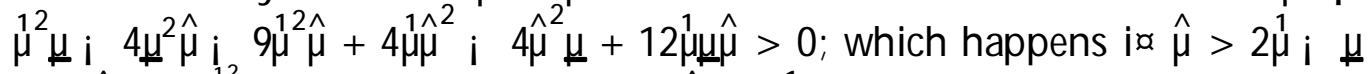
and $\hat{\mu}<\frac{\mu^{2}}{4\left(\hat{\mu}_{i} \downarrow\right)}$ : Notice however that $\hat{\mu}<2 \mu_{i} \underline{\mu}$ always. Hence $\epsilon_{1}>\varepsilon_{L}$ ix $\hat{\mu}<\frac{12}{4\left(\mu_{i} \downarrow\right)} ;$ that is the value $\hat{\mu}_{1}$ de. ned in the proposition.

To show part 1. of the proposition is suc ces to recall that it must be $C_{L}<E_{L}$ in order for piracy to be non-negative and that $i \max >\mid$ min for all $C_{L}<\varepsilon_{L}$ : We can therefore conclude $i \max >\mid$ min over the whole relevant range for $C_{L}$. Figure 1 illustrates this situation.

To show part 2. of the proposition, consider that $\hat{\mu}>\hat{\mu}_{1}$ implies $\epsilon_{\mathrm{L}}<\varepsilon_{\mathrm{L}}$; so that, for all $\underline{C}_{L}<C_{L}<\epsilon_{1},|\max >|$ min and for all $\epsilon_{L 1}<C_{L}<\varepsilon_{L}$; i max $<1$ min:This second case is illustrated by Figure 3 . 


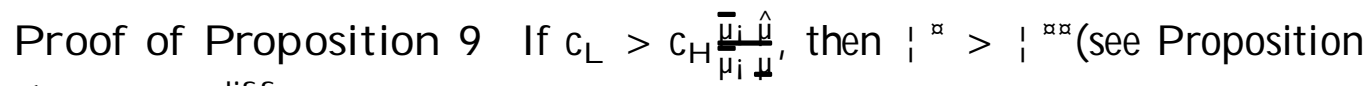
4). Then $!^{\text {dif } f}>\left.\right|^{x}$ ix

$$
\frac{c_{L} \hat{\mu}_{i} \underline{\mu}^{2}+c_{H_{3}}{ }^{3} \mu_{i} \hat{\mu}^{\prime 3}, 2 \hat{\mu}_{i} \underline{\mu}}{\mu_{i} \underline{\mu}^{2} 2 \hat{\mu}_{i} \underline{\mu}}>c
$$

that is ix

$$
c_{L} \hat{\mu}^{3} \hat{i}^{3}{ }^{3} 2 \hat{\mu}_{i} \underline{\mu}^{\prime}+c_{H}{ }^{3} \mu_{i} \hat{\mu}^{\prime 3} 2 \hat{\mu}_{i} \underline{\mu}>0
$$

Condition(70) is always true if $\hat{\mu}<\frac{\hat{\mu}}{2 \hat{\mu_{i}} \underline{ }}$. If instead $\hat{\mu}>\frac{\hat{\mu}}{2 \hat{\mu_{i}} \underline{\underline{\nu}}}$, it is satis.ed if and only if

$$
c_{L}<c_{H} \frac{\stackrel{\mu}{\mu}_{3} \hat{\mu} 2 \hat{\mu}_{i} \underline{\mu}}{\vec{\mu} 2 \hat{\mu}_{i} \underline{\mu} i \hat{\mu}^{2}}
$$

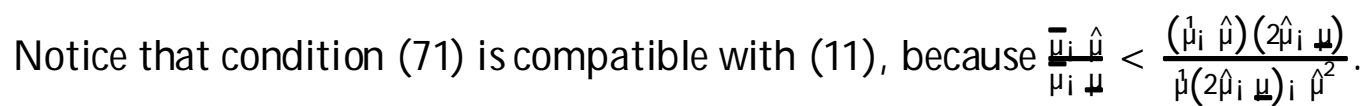
If instead $c_{L}<c_{H} \frac{\bar{\mu}_{i} \hat{\mu}}{\mu_{i} \underline{\underline{\mu}}}$, then ${ }^{x}>{ }^{x}$ (see Proposition 4 again). Then ! diff $>$ $i$

$$
\frac{c_{L} \hat{\mu}_{i} \underline{\mu}^{2}+c_{H_{3}}{ }^{3} \mu_{i} \hat{\mu}, 2 \hat{\mu}_{i} \underline{\mu}}{{ }^{1} \mu_{i} \underline{\mu}^{4} 2 \hat{\mu}_{i} \underline{\mu}}>c_{H} \frac{\mu_{i} \hat{\mu}}{\mu_{i} \underline{\mu}}
$$

which is always true. It then follows that under either equilibrium strategy piracy is eliminated.

Proof of Proposition 10 Comparing $\mid \min$ and $\mid{ }^{\text {" }}$ (given respectively by expressions (57) and (7)) we ...nd that $i$ min $>{ }_{i}^{a}$ when $\hat{\mu}<\frac{\underline{u}^{2}}{4\left(\hat{\mu}_{i} \underline{\mu}\right)}=\hat{\mu}_{1}$ : The strategy of producing one product and selling it to the whole market is never adopted in equilibrium. This implies that if $i \max >\mid \min$ for the relevant range of $c_{L}$ (which happens if $\hat{\mu}<\hat{\mu}_{1}$ ), then $\mid \min >{ }^{\text {x }}$ (see proposition 8).

We now prove that $\mid$ max crosses (pro..t from producing one good allowing piracy, given by expression (10)) once.

Comparing i max and ${ }^{\text {we see that } i \max <}$ for all $\mathrm{R}_{1}<\mathrm{C}_{\mathrm{L}}<\mathrm{R}_{2}$; where

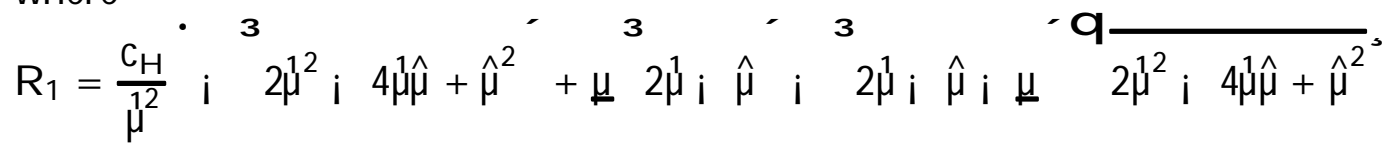
and

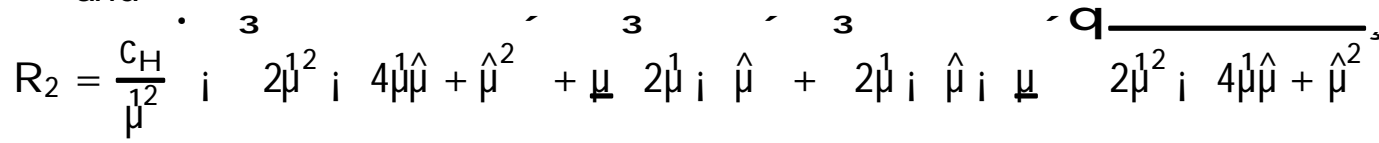


It is possiple to prove that $R_{1}<Q_{i}$ To check this see that $R_{1}<0$ for

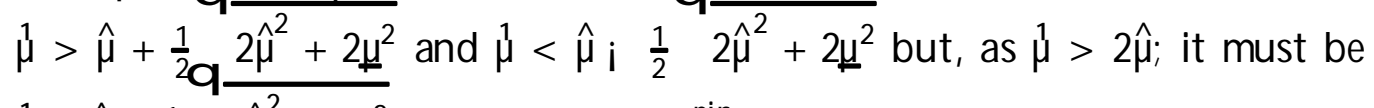

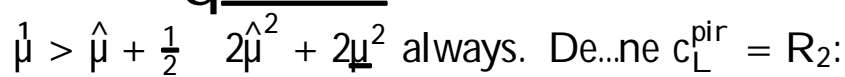

Then we show that $i \max$ crosses ${ }^{\infty}$ once for $C_{L}>\underline{C}_{L}$ and, for $C_{L}<C_{L}^{\text {pir }}$;

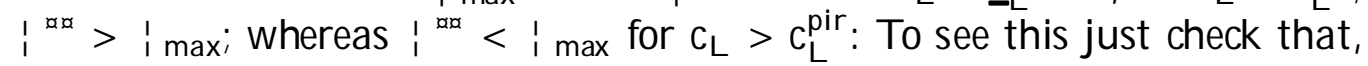
for $C_{L}=\underline{c}_{L} ;|\max <|$ a $:$ In fact,

$$
i^{i} i \max _{C_{L}=c_{L}}=\frac{c_{H} \mu^{3} \mu_{3}^{1}{ }^{1} 2 \hat{\mu}}{2^{i_{1}} \mu_{i} \underline{\mu}^{4} 3 \mu_{i} 2 \hat{\mu}}>0
$$

(recall that $\hat{\mu}>2 \hat{\mu}$ and is invariant with respect to $C_{L}$ ). We have shown in the proof to proposition 8 that 1 max is increasing and convex, so I max cuts I from below and is smaller than! for $\mathrm{C}_{\mathrm{L}}<\mathrm{C}_{\mathrm{L}}^{\text {pir }}$ and larger for $\mathrm{Q}>\mathrm{C}_{\mathrm{L}}^{\text {pir }}$ : However, while $c_{L}^{\text {pir }}>\underline{c}_{L}$ always, $c_{L}^{\text {pir }}<\mathcal{E}_{L}$ i $\hat{\mu}>\hat{\mu}_{2}$; where $\hat{\mu}_{2}=\frac{\hat{\mu}^{2} i 4^{2} \mu_{j} 4 \omega^{2}}{2\left(3 \mu_{i}^{2} 2 \mu\right)}$ : When $c_{L}^{\text {pir }}>\varepsilon_{L},\left.\right|^{\infty}>1$ max always in the relevant range of $c_{L}$ :

Recall that in part 1 . of the proposition $\hat{\mu}<\hat{\mu}_{1}$ : Therefore $c_{L}^{\text {pir }}<\xi_{\mathrm{L}}$ ix $\hat{\mu}_{2}<\hat{\mu}<\hat{\mu}_{1}$ : To have $\hat{\mu_{2}}<\hat{\mu}_{1}$ it must be that $\hat{\mu}>\hat{i}_{3}+{ }_{\overline{5}} \underline{\mu}$ : When this happens, we can have two cases. 1) $\hat{\mu}_{2}<\hat{\mu}<\hat{\mu}_{1}$, that is $\mathrm{C}_{\mathrm{L}}^{\mathrm{pir}}<\varepsilon_{\mathrm{L}}$ : In this case, we have shown that $! \max >1$ min when $\hat{\mu}<\hat{\mu}_{1}$ : We can then conclude that, for all $C_{L}<C_{L}^{\text {pir }} ; \mid>1 \max$ and for all $C_{L}>C_{L}^{\text {pir }} ; ! \max >\mid$ Figure 4 illustrates this situation. 2) $\hat{\mu}<\hat{\mu}_{2}<\hat{\mu}_{1}$ and $c_{L}^{\text {pir }}>\xi_{L}:$ In this case, the largest pro..t is : in the whofe relevant range.

Conversely, when $\hat{\mu}^{\prime}<3+\overline{5} \underline{\mu} ; c_{\mathrm{L}}^{\text {pir }}>\xi_{\mathrm{L}}$ and $\mathrm{m} \max <1$ in the whole relevant range.

To prove part 2., recall that when $\hat{\mu}>\hat{\mu}_{1} ;{ }^{x}>1$ min and $\epsilon_{1}<\varepsilon_{L}$ : Hence, $\varepsilon_{\mathrm{L}}$ is the largest solution to $\max _{\mathrm{m}}=\mathrm{m}$ min.

$i \max$ crosses $\left.\right|^{x}=a_{L}$ twice. Solve ${ }_{\max }=C_{L}$ and obtain two solutions

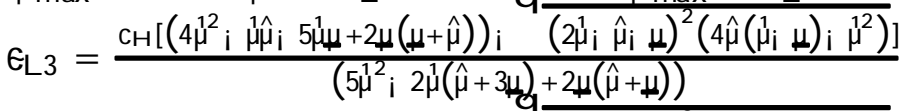

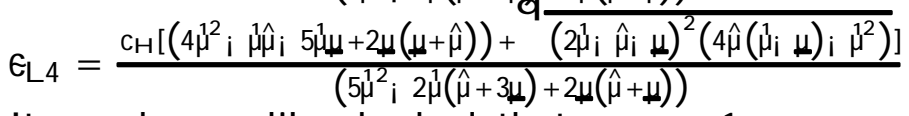

It can be readily checked that $\epsilon_{\mathrm{L}_{3}}<\xi_{\mathrm{L}}<\epsilon_{\mathrm{L} 4} ; \mathrm{M}$ oreover, $\mathrm{\epsilon}_{3}<\underline{\mathrm{C}}_{\mathrm{L}}$ :

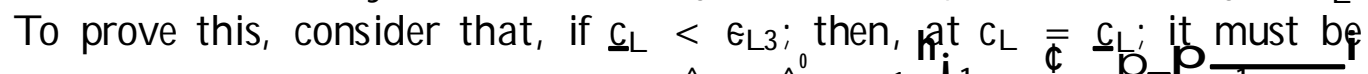

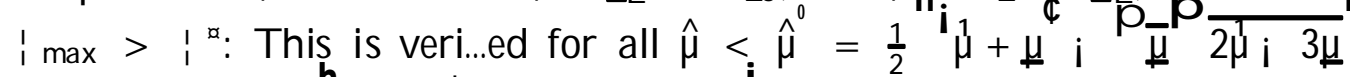
and $\hat{\mu}>\hat{\mu}^{\infty}=\frac{1}{2} h_{i_{1}} \mu+\underline{\mu}+{ }^{p} \underline{\underline{\mu}} p \frac{}{2 \mu_{i} 3 \underline{\mu}}$ : Considering that we are in the case where $\hat{\mu}<\frac{1}{2}$ (see Lemma 5) and that $\hat{\mu}<\frac{1}{2}<\hat{\mu}^{\infty}$ the relevant value 
is $\hat{\mu}$ : Check that $\hat{\mu}_{1}>\hat{\mu}$ always, so the only feasible case is $\hat{\mu}<\hat{\mu}<\frac{\mu}{2}$ (the other possible case, $\hat{\mu}<\hat{\mu}$ is ruled out by the fact that $\hat{\mu}_{1}>\hat{\mu}$ and we are considering $\left.\hat{\mu}>\hat{\mu}_{1}\right)$. Then, at $c_{L}=\underline{c}_{L} ; i \max <\mid{ }^{x}$ always and $\epsilon_{L_{3}}<\underline{c}_{L}$; Therefore in the relevant range for $\left.\underline{G}_{L}\left(\underline{c}_{L}<c_{L}<\xi_{L}\right)\right|^{\infty}>\mid$ min and ${ }^{\infty}>$ i max:

Since dixerentiation is never an optimal strategy in this case, the monopolist will produce one quality either by setting such a low price that piracy is eliminated or by setting a higher price and allowing some piracy. The latter strategy, which yields pro..ts ! corresponding pro..ts are ${ }^{x}$ ) if and only if $\mathrm{C}_{\mathrm{L}}<\mathrm{C}_{\mathrm{H}} \frac{\mathrm{l}_{\mathrm{H}} \hat{\mu}}{\mathrm{H}_{\mathrm{L}}}$ (see Proposition 4). This case is depicted in Figure 5.

Notice that $c_{H} \frac{\mu_{i} \hat{\mu}}{\mu_{i} \frac{\mu}{\underline{H}}}>\underline{c}_{L}$; therefore it is never the case that $\left.\right|^{\infty}>\left.\right|^{\infty}$ on the whole range $\left(\underline{c}_{L} ; \vec{\varepsilon}_{L}\right)$, whereas ${ }^{\prime}$ is overall greater than ${ }^{*}$ in the range $\left(\underline{c}_{L} ; \varepsilon_{L}\right)$ if $c_{H} \frac{\mu_{i} \hat{\mu}}{\mu_{i} \downarrow}>\varepsilon_{L}$; that is if $\hat{\mu}>\frac{\underline{h}^{2}+2 \mu^{2} \underline{\mu}_{i}}{2 \underline{u}_{\underline{L}}^{2}}$ :

\section{R eferences}

[1] Besen, S., and S. Kirby (1989), Private Copying, A ppropriability, and Optimal Copying R oyalties, J ournal of Law and Economics, 32, 255-280.

[2] Conner, K ., and R. Rumelt (1991), Software Piracy: An A nalysis of Protection Strategies, M anagement Science, 37, 125-139.

[3] Crampes, C., and A. Hollander (1995), Duopoly and Quality Standards, European Economic Review, 39, 71-82.

[4] Gabszewicz, J J., A. Shaked, J. Sutton, and J .-F. Thisse (1986), Segmenting the M arket: The M onopolist Optimal P roduct M ix, J ournal of E conomic Theory, 39(2), 273-289.

[5] Gayer, A., and O. Shy (2001), Internet, Peer-to-Peer, and Intellectual Property in Markets for Digital Products, mimeo, University of Haifa.

[6] Hui, K.L., Png, I.P.L., and Y. Cui (2001), Piracy and the Demand for Legitimate Recorded Music, mimeo, National University of Singapore, downoadable from http:// www.ssrn.com/.

[7] Itoh, M. (1983), Monopoly, Product Dixerentiation and Economic Welfare, J ournal of E conomic Theory, 31, 88-104.

[8] J ohnson, W. (1985), The Economics of Copying, J ournal of Political Economy, 93, 158-174. 
[9] Kahin, B., and H. Varian (eds.) (2000), Internet Publishing and Beyond: The E conomics of Digital Information and Intellectual Property. Cambridge, MA: T he MIT Press.

[10] Lambertini, L. (1997), T he Multiproduct Monopolist under Vertical Differentiation: An Inductive A pproach, Recherches E conomiques de Louvain, 63(2), 109-22.

[11] Liebowitz, S. ( 1985), Copying and Indirect A ppropriability: P hotocopying of J ournals, J ournal of Political E conomy, 93, 945-957.

[12] M askin E ., and J . Riley (1984), M onopoly with Incomplete Information, Rand J ournal of Economics, 15(2), 171-196.

[13] M otta, M . (1993), E ndogenous Qual ity Choice: Price vs. Quantity Competition, J ournal of Industrial E conomics, 41(2), 113-31.

[14] M ussa, E., and S. R osen (1978), M onopoly and P roduct Quality, J ournal of Economic Theory, 18, 171-196.

[15] Spence, M . (1975), Monopoly, Quality and R egulation, B ell J ournal of E conomics, 6, 417-429.

[16] Shaked, A ., and J . Sutton (1982), Relaxing Price Competition through Product Dixerentiation, Review of E conomic Studies, 49, 3-13.

[17] Shy, O. (2001), The Economics of Network Industries, Cambridge University Press.

[18] Shy, O., and J.-F. Thisse (1999), A Strategic Approach to Software Protection, J ournal of E conomics and Management Strategy, 8, 163-190.

[19] Tirole, J. (1988), The Theory of Industrial Organization, Cambridge, MA: MIT University Press.

[20] Varian, Hal R. (2000), Buying, Sharing and R enting Information G oods, J ournal of Industrial Economics, 48(4), 473-88. 